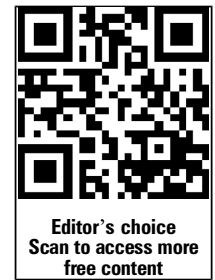

free content

\title{
Screening for interstitial lung disease in systemic sclerosis: performance of high-resolution CT with limited number of slices: a prospective study
}

\author{
Thomas Frauenfelder, ${ }_{1}^{1}$ Anna Winklehner, ${ }_{1}^{1}$ Thi Dan Linh Nguyen, ${ }^{1}$ \\ Rucsandra Dobrota, ${ }^{2,3}$ Stephan Baumueller, ${ }_{1}^{1}$ Britta Maurer, ${ }^{2}{ }$ Oliver Distler ${ }^{2}$
}

\section{Correspondence to} Dr Oliver Distler, Division of Rheumatology, University Hospital Zurich, Gloriastr. 25, Zürich 8091, Switzerland; Oliver.Distler@usz.ch

Received 31 March 2014 Revised 19 August 2014 Accepted 12 September 2014 Published Online First 30 September 2014

ABSTRACT

Objectives Early diagnosis of interstitial lung disease (ILD), currently the main cause of death in systemic sclerosis (SSc), is needed. The gold standard is highresolution $\mathrm{CT}$ (HRCT) of the chest, but regular screening faces the risk of increased radiation exposure. We performed a prospective validation of a dedicated, 9-slice HRCT protocol with reduced radiation dose for the detection of ILD in patients with SSC.

Methods We analysed 170/205 consecutive patients with SSc. Whole-chest HRCT, serving as standard of reference, and the reduced HRCT with nine slices allocated according to a basal-apical gradient were obtained. ILD presence, extent (> or $<20 \%$ ) and diagnostic confidence were assessed. The reduced HRCT was independently analysed by two blinded radiologists, who also evaluated image quality. Radiation dose parameters were calculated.

Results Standard chest HRCT showed ILD in 77/170 patients. With the reduced HRCT, 68/77 cases with ILD were identified (sensitivity $88.3 \%$, both readers). The accuracy $(91.8 \%$, reader $1 ; 94.7 \%$, reader 2$)$, diagnostic confidence $(98.8 \%$, reader $1 ; 95.3 \%$, reader 2 ) and image quality rates were high. Minimal ILD was correctly quantified in $73.1 \%$ (reader 1)/71.2\% (reader 2) and extensive ILD in $88 \%$ (reader 1)/100\% (reader 2). Importantly, the reduced HRCT had a significantly lower radiation dose. The mean dose length product (effective dose) was only $5.66 \pm 4.46 \mathrm{mGycm}(0.08 \pm 0.06 \mathrm{mSv})$ compared with the standard protocol dose of 149.00 $\pm 95.90 \mathrm{mGycm}(2.09 \pm 1.34 \mathrm{mSv})$.

Conclusions The above-described reduced chest HRCT protocol reliably detects even mild SSc-ILD in clinical practice, with the advantage of a much lower radiation dose compared with standard whole-chest HRCT.

CrossMark

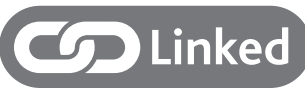

- http://dx.doi.org/10.1136/ annrheumdis-2014-206585

To cite: Frauenfelder $\mathrm{T}$, Winklehner A, Nguyen TDL, et al. Ann Rheum Dis 2014;73:2069-2073.

\section{INTRODUCTION}

Interstitial lung disease (ILD) is frequent in patients with systemic sclerosis (SSc) and is the leading cause of disease-related death. ${ }^{1}$ Nowadays, highresolution CT (HRCT) is the standard of reference for diagnosis of ILD. It allows an early detection of lung involvement, even from the subclinical stages. New methods enable a quantitative assessment and favour the use of HRCT for longitudinal studies. ${ }^{2-4}$

On the downside, CT uses ionising radiation, ${ }^{4}$ which has been linked to an increased cancer risk. $^{3} 5$ As patients with SSc are often screened annually, dose reduction is an important issue. ${ }^{67} \mathrm{In}$ the last decade, many methods have been introduced to lower the radiation dose by maintaining diagnostic image quality. Lowering tube current is the most widely employed approach in chest $\mathrm{CT}^{8}$ Alternatively, the number of slices can be reduced by performing a sequential CT protocol.

In an earlier, smaller, retrospective study, our group evaluated 'virtually' calculated series with a reduced number of slices for the detection and quantification of ILD. The results were promising, showing a high accuracy for the detection of SSc-ILD. ${ }^{9}$

The goals of this prospective study were therefore (1) to evaluate the robustness of the scan protocol concerning image acquisition; (2) to analyse the accuracy of a reduced chest scan with limited number of HRCT slices compared with a standard HRCT of the entire chest for the detection and quantification of ILD in patients with SSc and to validate thereby the results of the first retrospective study in a larger, prospective cohort and (3) to measure the reduction in radiation dose versus standard HRCT.

\section{METHODS}

\section{Patients' population}

A total of 205 consecutive patients with a diagnosis of SSc and annual follow-up in the Department of Rheumatology, University Hospital Zurich, were prospectively included in this study. All patients fulfilled either the American College of Rheumatology classification criteria ${ }^{10}$ or the Very Early Diagnosis Of Systemic Sclerosis (VEDOSS) criteria for SSc. ${ }^{11}$ Assessment of clinical parameters and image acquisition was done prospectively following international standards. ${ }^{12}$ All patients signed informed consent. The study was approved as part of the Zurich cohort of the European Scleroderma Trial and Research registry by the institutional review board.

\section{Image acquisition}

All patients were examined between January 2012 and September 2013. CT scans were performed with a 64-slice multidetector CT (Somatom Definition AS, Siemens Healthcare, Erlangen, Germany) or a 128-slice multidetector CT (Definition Flash Dual Source, Siemens Healthcare, Germany). Patients were examined in prone position and at end inspiration. Standard HRCT of the entire chest was performed and considered the standard of reference (tube potential $120 \mathrm{kV}$, reference tube current-time product 40 or $110 \mathrm{mAs}$, care dose on). Data were 
reconstructed with filtered back projection (FBP), a slice thickness of $1 \mathrm{~mm}$, an increment of $0.8 \mathrm{~mm}$, using a sharp (B60f) or very sharp convolution kernel (B70f).

Furthermore, a low-sampling HRCT of the chest with nine sequential slices, the so-called 'reduced HRCT', was obtained (tube potential $120 \mathrm{kV}$, reference tube current-time product 30 or $110 \mathrm{mAs}$, care dose on). The first three slices had an increment of $80 \mathrm{~mm}$ and were placed as follows: the first slice at the manubrium sterni, the second at the carina and the third at the lower lobe. For the following six basal slices, the increment was $15 \mathrm{~mm}$ (figure 1). Due to technical reasons, the CT scanner performed two slices per level. Data were reconstructed with FBP, a slice thickness of $1 \mathrm{~mm}$ and a very sharp (B70f) or ultrasharp convolution kernel (B80f).

\section{Image assessment}

The readout was performed on dedicated PACS-Viewer (Impax 6.4, AGFA, Dübendorf, Switzerland) and licensed reading screens. The readers were allowed to use all functions without multiplanar reformation, including changing window and level setting. All images were displayed on lung window.

On standard HRCT of the entire chest, presence of ILD (yes/ no; for ILD definition, see online supplementary text) was assessed by one reader experienced with chest CT (TF, 12 years of experience) as previously described. ${ }^{9}$ The extent of ILD was graded as no lung involvement, lung involvement less than $20 \%$ (i.e. limited disease), lung involvement more than $20 \%$ (i.e. extensive disease) or indeterminate (i.e. extent not readily classifiable) as described by Goh et al. ${ }^{4}$ In cases of uncertainty concerning the presence of ILD, the CT scans from previous annual follow-ups were taken into account. Degree of diagnostic confidence was recorded (score 1 to 4 ; 1 =fully confident; 2 =probably confident; $3=$ confident only under limited conditions; $4=$ not confident). ${ }^{13}$ Before the analysis of the reduced HRCT scans, all three readers underwent a training session including assessment and discussion of 10 non-study-related cases.

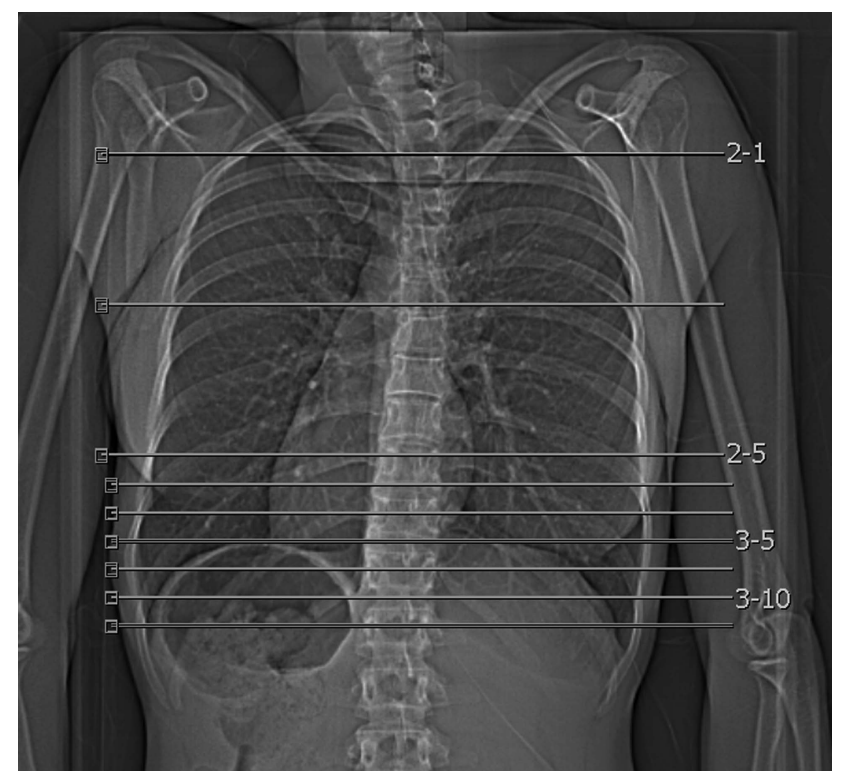

Figure 1 Topogram displaying the levels of high-resolution CT scans with a reduced number of slices. One image apical, one at the level of the carina and six images at the basal level with $1 \mathrm{~mm}$ slices, the upper three images with an increment of $80 \mathrm{~mm}$, the basal six images with an increment of $15 \mathrm{~mm}$.
The reduced HRCT scans were evaluated in a blinded manner, without knowing the results of the standard HRCT, by two radiologists (AW, 4 years of experience; SB, 6 years of experience). Again, presence and extent of ILD and the related diagnostic confidence were assessed. Furthermore, the quality of the image acquisition of reduced HRCT scans was rated on a scale from 1 to 3 as follows: $1=$ excellent (all slices contain lung tissue, base of the lung covered, second slice less than $2 \mathrm{~cm}$ above or below the level of the carina); 2 =acceptable (1-2 slices without capture of lung tissue and/or second slice more than $2 \mathrm{~cm}$ above or below the level of the carina); and $3=$ not acceptable (three or more slices without capture of lung tissue and/or second slice more than $3 \mathrm{~cm}$ above or below the level of the carina).

\section{Radiation dose}

Radiation dose parameters of standard HRCT of the entire chest and reduced HRCT were assessed from the patient protocol. Effective radiation dose in millisievert was estimated by multiplying the dose length product (DLP) with the region-specific conversion coefficient (chest $0.014 \mathrm{mSv} / \mathrm{mGycm}){ }^{14}$

\section{Statistical analysis}

Statistical analysis was performed using SPSS (SPSS, release V.21.0 for Windows; SPSS, Chicago, Illinois, USA). Continuous variables were reported as mean $\pm \mathrm{SD}$, and categorical variables as frequencies or percentages. Cohen's $\kappa$ statistics or Kendall's $\tau$ test was calculated for interobserver agreements as appropriate. Presence of ILD was compared with Cochrane's Q test and McNemar test. Sensitivity, specificity, accuracy and negative predictive value were calculated concerning the detection of ILD. The CI was 95\%. Statistical significance was inferred at a $\mathrm{p}$ value below 0.05 .

\section{RESULTS}

\section{Patients' demographics}

Of the 205 patients with SSc included, 35 could not be analysed (HRCT of the entire chest not available, $n=11$; image reconstruction with iterative reconstruction and not FBP, $n=16$; no available image protocol, $n=6$; reduced HRCT acquired with more than nine slices, $n=2)$. Thus, CT scans of 170 patients with SSc were assessed in this study (139 women, 31 men; median age $56(\mathrm{Q} 1, \mathrm{Q} 3=48,65.75))$. Clinical characteristics of these 170 patients with SSc are summarised in table 1.

\section{Image acquisition}

CT images were acquired with a tube potential of $120 \mathrm{kV}$ in all patients. The reference tube current-time product was $110 \mathrm{mAs}$ in standard HRCT and reduced HRCT in 72 patients $(42.4 \%)$, and $40 \mathrm{mAs}$ in standard HRCT and $30 \mathrm{mAs}$ in reduced HRCT in the remaining 98 patients $(57.6 \%)$. Both standard and reduced HRCT were reconstructed with a very sharp convolution kernel (B70f) in 89 patients (52.4\%), whereas in 66 patients $(38.8 \%)$ standard HRCT was reconstructed with a very sharp convolution kernel (B70f) and reduced HRCT with an ultrasharp convolution kernel (B80f). In 15 patients (8.8\%), a sharp convolution kernel was used in standard HRCT (B60f) and a very sharp convolution kernel in reduced HRCT (B70f).

\section{Image assessment}

Inter-reader agreement for reduced HRCT was good for the assessment of quality of image acquisition $(\kappa=0.71)(\tau=0.78)$ and for the analysis of extent of ILD $(\kappa=0.76)(\tau=0.84)$, and it 
Table 1 Characteristics of the study population

\begin{tabular}{|c|c|c|c|c|c|c|c|}
\hline $\begin{array}{l}\text { Age } \\
\text { (years) }\end{array}$ & $\operatorname{Sex}(n)$ & $\begin{array}{l}\text { Disease } \\
\text { duration* } \\
\text { (months) }\end{array}$ & Disease subsets (n) & mRSS* & $\begin{array}{l}\text { Antibody profile } \\
\text { (n of positives) }\end{array}$ & $\begin{array}{l}\text { Lung function } \\
\text { parameters (n) }\end{array}$ & $\begin{array}{l}\text { Major organ } \\
\text { involvement except } \\
\text { ILD }\end{array}$ \\
\hline $56(48,66)$ & $\begin{array}{l}\text { Men } n=31 / 170 \\
\text { Women } n=139 / \\
170\end{array}$ & $60(28,120)$ & $\begin{array}{l}\text { IcSSc }(n=66 / 166) \\
\text { dcSSc }(n=34 / 166) \\
\text { early/sine } \\
\text { scleroderma } \\
(n=66 / 166)\end{array}$ & $\begin{array}{l}\text { IcSSc: } 4(2,7) \\
\text { dcSSc:13.5 }(11,19) \\
\text { early/sine } \\
\text { scleroderma } 0 \\
(0,0)\end{array}$ & $\begin{array}{l}\text { ANA } n=163 / 170 \\
\text { Scl-70 } n=35 / 166 \\
\text { ACA } n=73 / 166 \\
\text { RNA polymerase } \\
\text { III } n=11 / 160 \\
\text { U1-RNP } n=7 / 164 \\
\text { Pm-SCl } n=13 / 149\end{array}$ & $\begin{array}{l}\text { DLCO }<70 \% \text { of } \\
\text { predicted } n=43 / 168 \\
\text { FVC }<70 \% \text { of } \\
\text { predicted } n=11 / 168 \\
\text { TLC }<70 \% \text { of } \\
\text { predicted } n=13 / 168\end{array}$ & $\begin{array}{l}\text { Renal crisis } n=3 / 167 \\
\mathrm{GI} \text { involvement } \\
n=109 / 168 \\
\text { Heart involvement } \\
n=60 / 167 \\
\text { PAH } n=7 / 170\end{array}$ \\
\hline
\end{tabular}

Disease duration: time from first non-Raynaud symptom (missing data $n=3$ ).

$\mathrm{Gl}$ involvement was defined as presence of symptoms of GI dysmotility.

Heart involvement was defined as presence of at least one of systolic/diastolic dysfunction on echocardiography, conduction block/significant arrhythmia on ECG.

PAH was defined as mPAP $\geq 25 \mathrm{~mm} \mathrm{Hg}$ at rest and PWP $\leq 15 \mathrm{~mm} \mathrm{Hg}$ at RHC; RHC was performed when PH was suspected by the treating physician (29/170 patients).

*Median (Q1, Q3).

ACA, anticentromere antibodies; ANA, antinuclear antibodies; dcSSC, diffuse cutaneous SSc; DLCO, diffusing capacity of the lung for carbon monoxide; FEV1, forced expiratory volume in

$1 \mathrm{~s}$; FVC, forced vital capacity; GI, gastrointestinal; ILD, interstitial lung disease; IcSSc, limited cutaneous SSC; mPAP, medium pulmonary artery pressure (RHC); PAH, pulmonary artery

hypertension; PH, pulmonary hypertension; Pm-Scl, anti-Pm-Scl antibodies; PWP, pulmonary wedge pressure; RHC, right heart catheterisation; RNA polymerase III, anti-RNA polymerase

III antibodies; SCl-70, antitopoisomerase 1 antibodies; SSC, systemic sclerosis; TLC, total lung capacity; U1-RNP, antiribonucleoprotein antibodies.

was excellent for the detection of ILD $(\kappa=0.82)$, as well as concerning diagnostic confidence $(\kappa=0.89)(\tau=0.94)$.

Quality of image acquisition of reduced HRCT scans was rated as excellent or acceptable in $93.5 \%(\mathrm{n}=159)$ by both readers (reader 1: 112 excellent/47 acceptable/11 not acceptable; reader 2: 96 excellent/63 acceptable/11 not acceptable).

ILD was present in $45.3 \%$ of subjects $(n=77 / 170)$ in standard HRCT. ILD was correctly diagnosed in $88.3 \%(n=68 / 77)$ of reduced HRCT scans, with a sensitivity of $88.3 \%$ for both readers and an accuracy of $91.8 \%$ (reader 1) and 94.7\% (reader 2 ), as shown in table 2 . In two patients, both readers missed the presence of ILD due to subtle changes located in the costodiaphragmatic sinus, which was not captured in the reduced HRCT scans, although they were classified as having excellent image quality (figure 2). In the remaining seven cases, the (minor) findings on reduced HRCT were interpreted as dystelectasis.

Diagnostic confidence was very high in standard HRCT (98.8\% fully confident, $1.2 \%$ probably confident) and high in reduced HRCT (reader 1: 91.2\% fully confident, 7.6\% probably confident, $1.2 \%$ confident only under limited conditions; reader $2: 70.0 \%$ fully confident, $25.3 \%$ probably confident, $4.7 \%$ confident only under limited conditions; $\mathrm{p}<0.05$ vs. standard HRCT, both readers). No reader felt 'not confident' (score 4) concerning the diagnosis of presence or absence of ILD in reduced or standard HRCT.

In patients with evidence of ILD $(n=77 / 170)$, the extent of ILD was estimated as minimal (i.e. $<20 \%$ ) in $52 / 77$ patients and as extensive (i.e. $>20 \%$ ) in $25 / 77$ patients in standard HRCT.

Table 2 Estimated accuracy and diagnostic certainty in detecting ILD on reduced HRCT scans

\begin{tabular}{lll}
\hline & Reader 1 & Reader 2 \\
\hline Sensitivity $(95 \% \mathrm{Cl})$ & $88.3 \%(78.5 \%$ to $94.2 \%)$ & $88.3 \%(78.5 \%$ to $94.2 \%)$ \\
Specificity $(95 \% \mathrm{Cl})$ & $94.6 \%(87.3 \%$ to $98.0 \%)$ & $100 \%(95.1 \%$ to $100 \%)$ \\
Accuracy & $91.8 \%$ & $94.7 \%$ \\
NPV $(95 \% \mathrm{Cl})$ & $90.7 \%(82.7 \%$ to $95.4 \%)$ & $91.2 \%(83.5 \%$ to $95.6 \%)$ \\
High diagnostic confidence* & $98.8 \%$ & $95.3 \%$ \\
\hline
\end{tabular}

*Degree of confidence score 1 or 2 (i.e. 1 =fully confident; 2 =probably confident). HRCT, high-resolution CT; ILD, interstitial lung disease; NPV, negative predictive value.
From the patients with minimal involvement on standard HRCT, 73.1\% (reader 1) and 71.2\% (reader 2) were classified correctly on reduced HRCT, $17.3 \%$ (both readers) were wrongly classified as no ILD and 9.6\% (reader 1) and $11.5 \%$ (reader 2) were overestimated. Regarding patients classified as

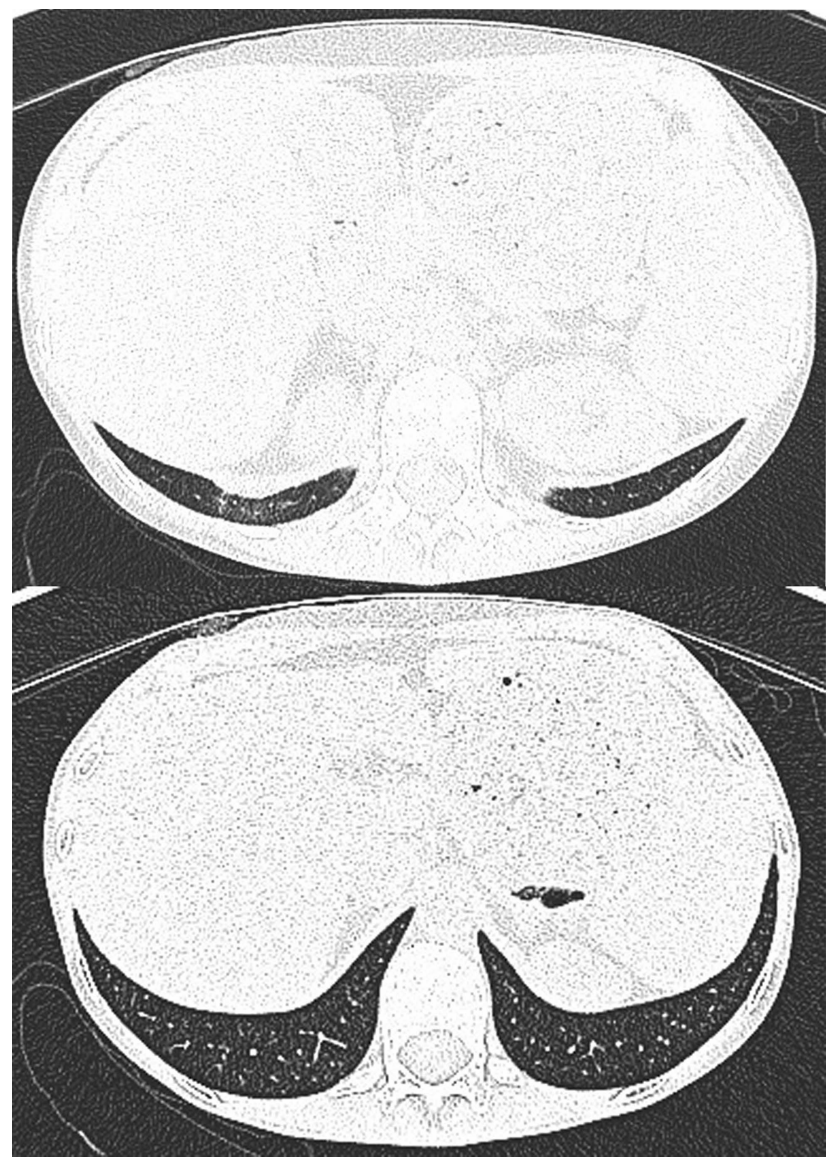

Figure 2 A female patient (35 years) with minimal interstitial lung disease missed by both readers in the reduced high-resolution CT (HRCT) scan. (Top) Transverse image of standard HRCT at basal level showing slight changes at the costodiaphragmatic sinus. (Bottom) The ninth most basal slice of the reduced HRCT scan, not capturing parenchymal changes. 
extensive in standard HRCT, 88\% (reader 1) and 100\% (reader 2) were correctly classified as extensive in reduced HRCT (table 3). All misclassified cases were also borderline on standard HRCT.

\section{Radiation dose}

In standard HRCT, mean overall DLP was 149.00 $\pm 95.90 \mathrm{mGycm}$ with an estimated effective radiation dose of $2.09 \pm 1.34 \mathrm{mSv}$, while in reduced HRCT, the mean overall DLP was $5.66 \pm 4.46 \mathrm{mGycm}$ with an effective radiation dose of 0.08 $\pm 0.06 \mathrm{mSv}(\mathrm{p}<0.001)$, resulting in an overall radiation dose reduction of $96.2 \%$.

\section{DISCUSSION}

This prospective study evaluated a dedicated HRCT protocol consisting of only nine slices with a basal-apical gradient for the detection of ILD in patients with SSc. Our results show that this protocol has a high accuracy and sensitivity in the detection of SSc-ILD, significantly reduces the radiation dose and can be implemented in daily routine.

Screening and early detection of ILD in patients with SSc is important as pulmonary involvement is a negative prognostic factor. Goh et $a l^{4}$ showed that interstitial changes extending more than $20 \%$ on HRCT are associated with a higher mortality. Incipient lung changes like ground glass opacities are very subtle, cannot be visualised on chest radiography and do not lead to a quantifiable decrease in lung function parameters. They are, nonetheless, prone to progress, and their early detection might allow early access to treatment and, hopefully, a better outcome. Without question, early diagnosis of SSc-ILD is, due to the leading role of lung involvement in SSc-related mortality, a priority, and HRCT is the most sensitive method. However, just like the use of HRCT for early lung cancer detection, the use of annual HRCT as a screening method for ILD has to be considered carefully because of the cumulative radiation dose. It is assumed that patients might be especially exposed to the attributable risk for developing cancer after repetitive CT-generated ionising radiation. ${ }^{67}$ Based on the principle of As Low As Reasonably Achievable, all possible methods to reduce radiation dose by maintaining diagnostic image quality have to be applied. ${ }^{15} 16$ When compared with early lung cancer detection programmes, the use of low-dose or ultra-low-dose CT is limited as the current tube dose reduction leads to an increase in noise and makes the detection of subtle interstitial findings impossible. We therefore investigated a different method by reducing the number of CT slices per scan. Indeed, using our protocol, the radiation dose could be lowered down to $0.08 \mathrm{mSv}$, which is a meaningful dose reduction of $96.2 \%$, to a level comparable with a conventional chest radiograph $(0.05 \mathrm{mSv})$. This is an important advancement in the strategy to reduce radiation exposure, while at the same time improving early and sensitive detection of lung involvement in patients with SSc (see online supplementary text).

In a previous retrospective study, ${ }^{9}$ our group evaluated the use of such a protocol by retrospectively extracting the relevant slices from a whole-chest HRCT of patients with SSc. Different image series were evaluated. The best performance, with equal results to standard HRCT, was an image series with a basalapical gradient, consistent with the mainly basally located parenchymal changes in ILD. ${ }^{2}$ Based on these results, the HRCT protocol used in the current study was designed. This protocol differs slightly from the one in the retrospective study due to technical and user-related reasons. As scans were acquired by CT technicians with different training levels and different experience, the protocol had to be easily usable, with minimal variables. Our results show that the majority of exams were classified as excellent and acceptable. The most frequently reported fault was related to the most basal slices, which were scanned either too low or slightly too high, the latter leading to false negative results.

Concerning the detection of ILD, the results showed a high accuracy and sensitivity of $93 \%$ and $88.3 \%$, respectively, only slightly below the values from the retrospective study. False negative results were mainly due to small areas of ILD in the costodiaphragmatic angle, which is likely not clinically meaningful. There were few false positive results caused by misinterpretation of noise as ground glass opacities.

Visual assessment of the extent of fibrosis with the reduced HRCT protocol was good in extensive disease and satisfactory in minimal disease, in which case $17.3 \%$ of cases were missed, and $9.6 \%$ (reader 1 ) and $11.5 \%$ (reader 2 ) were overestimated.

This limitation can be overcome through alternative methods for the assessment of the extent of ILD. A well-described option is the use of CT densitometric parameters like skewness and kurtosis of the CT histograms of the lung tissue. ${ }^{17}$ These parameters have been validated by various authors for the whole-chest CT and correlate with spirometric lung function parameters. ${ }^{17-19}$ Initial results from an ongoing study with the same population show that this method can be translated into our protocol and enables the quantification of the reduced HRCT. This would also be significant with regard to the implementation of such a protocol for follow-up exams, where smaller changes have to be expressed quantitatively.

Our study has the following limitations: HRCT protocols used different tube current-time product and convulsions kernels for the standard whole-chest HRCT and the reduced HRCT between exams. As there was no effect on the detection of fibrosis, we did not exclude these cases from the analysis. The reason for the different kernels was that the kernels provided by the scanner were not identical for sequence and spiral

Table 3 Estimated extent of ILD in standard HRCT and reduced HRCT scans

\begin{tabular}{|c|c|c|c|c|}
\hline \multirow[b]{2}{*}{ Lung involvement in standard HRCT } & \multicolumn{4}{|c|}{ Estimated extent in reduced HRCT } \\
\hline & $\begin{array}{l}\text { No ILD } \\
\text { Reader } 1 / 2\end{array}$ & $\begin{array}{l}\text { Minimal }(<20 \%) \\
\text { Reader } 1 / 2\end{array}$ & $\begin{array}{l}\text { Extensive (>20\%) } \\
\text { Reader } 1 / 2\end{array}$ & $\begin{array}{l}\text { Indeterminate } \\
\text { Reader } 1 / 2\end{array}$ \\
\hline $\begin{array}{l}\text { Minimal }(<20 \%) \\
\mathrm{n}=52\end{array}$ & $n=9 / 9$ & $\mathrm{n}=38 / 37^{*}$ & $n=5 / 6$ & $\mathrm{n}=0 / 0$ \\
\hline $\begin{array}{l}\text { Extensive }(>20 \%) \\
n=25\end{array}$ & $\mathrm{n}=0 / 0$ & $n=3 / 0$ & $n=22 / 25^{*}$ & $\mathrm{n}=0 / 0$ \\
\hline
\end{tabular}


mode. Another limitation is represented by the asymmetric distribution of slices according to the basal-apical gradient, with a wide gap of non-depicted lung in the mid and upper parts. To further improve estimation of the extent of lung involvement, some additional slices in these parts might be necessary. Furthermore, slight fibrotic changes at the level of the costodiaphragmatic sinus can be missed in the reduced HRCT protocol due to lack of coverage, although the clinical meaning of these subtle changes might be questionable. In addition, the primary focus being ILD, other potentially relevant findings can be missed (see online supplementary text), making the reduced HRCT perhaps best for younger patients, less likely to have important incidental findings. Finally, future studies in clinical practice have to show whether the new HRCT protocol can replace conventional HRCT protocols or whether a combination of protocols, for example, by the use of reduced HRCT for screening and conventional HRCT for confirmation of potentially meaningful changes is more accurate.

In conclusion, our study shows that a dedicated, reduced HRCT protocol consisting of nine slices allocated according to a basal-apical gradient represents a robust and accurate method to reliably detect ILD in patients with SSc. With the considerable advantage of a low radiation dose, this study provides evidence that it can be implemented in daily clinical routine for early detection and screening of ILD.

Contributors TF designed the study, analysed HRCTs, interpreted and analysed the data and wrote the manuscript. AW analysed HRCTs, interpreted and analysed the data and wrote the manuscript. TDLN and SB analysed HRCTs and interpreted and analysed the data. RD interpreted and analysed the data and wrote the manuscript. BM obtained clinical data, interpreted and analysed the data. OD designed the study, obtained clinical data, interpreted and analysed the data and wrote the manuscript.

Funding RD's research activity was supported by an Articulum Fellowship (2013-2014), an EULAR training bursary and a research grant from Actelion Pharmaceuticals. All other authors were supported by their institutions.

Competing interests OD has/had consultancy relationship and/or has received research funding in the area of SSc and related conditions from Actelion, Pfizer, Ergonex, BMS, Sanofi-Aventis, United BioSource Corporation, Roche/Genentech, Medac, Biovitrium, Boehringer Ingelheim Pharma, Novartis, 4 D Science, Active Biotec, Bayer-Schering, Sinoxa, Serodapharm, EpiPharm, Biogen, Inventiva and GSK. The real or perceived potential conflicts listed above are accurately stated.

Patient consent Obtained.

Ethics approval The study was approved by the University Hospital Zurich Institutional Review Board.

Provenance and peer review Not commissioned; externally peer reviewed.

\section{REFERENCES}

1 Steen VD, Medsger TA. Changes in causes of death in systemic sclerosis, 1972-2002. Ann Rheum Dis 2007;66:940-44.

2 Strollo D, Goldin J. Imaging lung disease in systemic sclerosis. Curr Rheumatol Rep 2010;12:156-61.

3 Kaloudi 0 , Miniati I, Alari S, et al. Interstitial lung disease in systemic sclerosis. Intern Emerg Med 2007;2:250-5.

4 Goh NS, Desai SR, Veeraraghavan S, et al. Interstitial lung disease in systemic sclerosis: a simple staging system. Am J Respir Crit Care Med 2008;177: 1248-54.

5 Brenner DJ, Hall EJ. Computed tomography-an increasing source of radiation exposure. N Engl J Med 2007;357:2277-84.

6 Sodickson A, Baeyens PF, Andriole KP, et al. Recurrent CT, cumulative radiation exposure, and associated radiation-induced cancer risks from CT of adults. Radiology 2009;251:175-84.

7 Amis ES Jr, Butler PF. ACR white paper on radiation dose in medicine: three years later. J Am Coll Radiol 2010;7:865-70.

8 Kubo T, Lin PJ, Stiller W, et al. Radiation dose reduction in chest CT: a review. Am J Roentgenol 2008;190:335-43.

9 Winklehner A, Berger N, Maurer B, et al. Screening for interstitial lung disease in systemic sclerosis: the diagnostic accuracy of HRCT image series with high increment and reduced number of slices. Ann Rheum Dis 2012;71:549-52.

10 Subcommittee for scleroderma criteria of the American Rheumatism Association Diagnostic and Therapeutic Criteria Committee. Preliminary criteria for the classification of systemic sclerosis (scleroderma). Arthritis Rheum 1980;23:581-90.

11 Avouac J, Fransen J, Walker UA, et al. Preliminary criteria for the very early diagnosis of systemic sclerosis: results of a Delphi Consensus Study from EULAR Scleroderma Trials and Research Group. Ann Rheum Dis 2011:70:476-81.

12 Walker UA, Tyndall A, Czirjak L, et al. Clinical risk assessment of organ manifestations in systemic sclerosis: a report from the EULAR Scleroderma Trials And Research group database. Ann Rheum Dis 2007;66:754-63.

13 Prakash P, Kalra MK, Kambadakone AK, et al. Reducing abdominal CT radiation dose with adaptive statistical iterative reconstruction technique. Invest Radiol 2010;45:202-10

14 Medicine AAoPi. The measurement, reporting, and management of radiation dose in CT. Report of AAPM Task Group 23 of the Diagnostic Imaging Council CT Committee; 2008. http://www.aapm.org/pubs/reports/rpt_96.pdf (accessed Jan 2014).

15 Picano E, Matucci-Cerinic M. Unnecessary radiation exposure from medical imaging in the rheumatology patient. Rheumatology (Oxford) 2011;50:1537-39.

16 Niemann T, Zbinden I, Roser HW, et al. Computed tomography for pulmonary embolism: assessment of a 1-year cohort and estimated cancer risk associated with diagnostic irradiation. Acta Radiol 2013;54:778-84.

17 Best AC, Lynch AM, Bozic CM, et al. Quantitative CT indexes in idiopathic pulmonary fibrosis: relationship with physiologic impairment. Radiology 2003;228:407-14.

18 Camiciottoli G, Orlandi I, Bartolucci $M$, et al. Lung CT densitometry in systemic sclerosis: correlation with lung function, exercise testing, and quality of life. Chest 2007:131:672-81.

19 Sverzellati N, Zompatori M, De Luca G, et al. Evaluation of quantitative CT indexes in idiopathic interstitial pneumonitis using a low-dose technique. Eur J Radiol 2005:56:370-5. 